\title{
Nanowire gate all around-TFET-based biosensor by considering ambipolar transport
}

\author{
N. Nagendra Reddy ${ }^{1} \cdot$ Deepak Kumar Panda ${ }^{1}$ (D)
}

Received: 13 May 2021 / Accepted: 9 August 2021 / Published online: 19 August 2021

(c) The Author(s), under exclusive licence to Springer-Verlag GmbH, DE part of Springer Nature 2021

\begin{abstract}
This work investigated the performance of overlapped gate-on-drain of a gate all around-tunnel field-effect transistor (GAATFET) biosensors by considering the dielectric modulated technique by immobilizing the targeted biomolecules in the cavity region curved under the overlapped gate-on-drain. The nanowire GAA-TFET device shows excellent controllability over the channel and reduces leakage current to a greater extent. Here, we tried to make the ambipolar nature of the TFET, an advantage for the biosensor by detecting the biomolecule using variation of ambipolar current of TFET. Due to structural arrangement, the nanocavity under the overlapped gate region suppresses the ambipolar drain current by increasing the dielectric constant of the targeted biomolecules. The device can show a variation of $10^{2}$ and $10^{3}$ amount of sensitivity for the variation of dielectric constant from 1 to 5 and, compared with the other TFET structure, the proposed overlapped gateon-drain GAA-TFET biosensor shows higher sensitivity and low leakage with a highly controlled channel.
\end{abstract}

Keywords Ambipolar behavior · Biosensor · Dielectric Modulation (DM) · Tunnel Field-Effect Transistor (TFET) · Gate All Around-Tunnel Field-Effect Transistor (GAA-TFET) · Overlapping gate-on-drain

\section{Introduction}

At dawn, and evaluation of the first-ever developed biosensor gives everyone optimism that there is an alternative to traditional laboratory techniques for detecting biomolecules $[1,2]$. The stupendous characteristics of biosensors created enormous interest to carry forward the research in this field of biosensors. The advancement in the technology helped to drift the biosensor field to the extreme level and spread their application to many areas like environment monitoring, food processing, and the public health care sector. The biosensor plays an essential role in the medical field because of the following characteristics: early detection, continuous monitoring, and disease diagnosis. The present corona (Covid-19) pandemic's situation is the best indication of biosensor's importance in medical diagnosis since the early identification of the disease can stop the virus's outbreak transmission from one to many [1]. Therefore, with the biosensor's

Deepak Kumar Panda

deepakiitkgp04@gmail.com

1 Microelectronics and VLSI Design Group, School of Electronics, VIT-AP University, Amaravati, Andhra Pradesh 522237, India aid, they can carry out tests, trace, isolate, and prevent form transmission of disease.

Based on the kind of techniques, such as bio-recognition and identification methodologies, the biosensors are classified. The biosensor has divided into two types based on the detection technique: (1) label-based detection and (2) label-free detection. Due to its simple and costeffective design approaches, the label-free detection technique has gained tremendous popularity. The field-effect transistor (FET)-based biosensors created revolutionary change and development in the field of biosensors [2-7]. The electrical detection of the target biomolecules using FET by virtue of label-free detection can develop a biosensor with the following characteristics (1) high sensitivity, (2) high selectivity, (3) On-chip integration of both sensor and the amplifier (4) Cost-effective and (5) Possibility of mass production and Reusability. Many researchers were impressed and inspired by the FET-based biosensors' characteristics, carried out immense research, and reported a good and fruitful development in FET-based biosensors. The performance of the FET-based biosensors is high in class, but still, they are beyond touching the tip of the sensitivity because of the following hectic issues (1) the theoretical constraint on the minimum achievable 
subthreshold slope ( $\mathrm{SS}>60 \mathrm{mv} / \mathrm{dec}$ ) due to Boltzmann tyranny $[\mathrm{KBT} / \mathrm{q} \ln (10)]$ and (2) the short channel effects experience by the FET device in the process of curtailing and scaling [8].

The transistor's scaling results in the reduction of device size, operating voltages, and density on the chip. This scaling results in the reduction of gate length as a counteraction, we have to reduce the threshold voltage $\left(V_{\mathrm{th}}\right)$, and the supply voltage $\left(V_{\mathrm{DD}}\right)$ of the transistor needs to be scaled down to maintain overdrive as high as possible $\left(V_{\mathrm{DD}}-V_{\mathrm{th}}\right)$. This action results in the exponential raising of the leakage current of the transistor. We have to scale down the transistor's subthreshold swing to maintain the stability between the overdrive and the leakage current. The scaling of the FET increases the amount of heat generated for every switching process, leading to high power dissipation and shows an impact on device performance [8]. The power dissipation of the FET is in control when it is operating at the lower voltage, but the limitation on the subthreshold swing does not allow the conventional FET (CFET) to operate at lower voltages.

In recent times, the tunnel field-effect transistor (TFET) has become a prominent substitute to the CFET by employing a distinct and unique carrier transport mechanism bandto-band tunneling form source to channel with a minimal gate voltage [8-10]. The TFET can exhibit a superior subthreshold swing ( $\mathrm{SS}<60 \mathrm{mv} / \mathrm{dec}$ ), which is the key performance metric for designing highly efficient biosensors. Many researchers successfully reported their research in the TFET-based biosensors in many articles with enhanced techniques to bring out the high sensitivity. The ambipolar conductivity and the low on current $\left(I_{\mathrm{ON}}\right)$ are two downsides of the TFET and become an obstacle to develop biosensors with high performance and sensitivity. However, researchers are trying to expose the downside of the TFET as an advantage for sensing, but there is still a gap to accomplish a high and efficient biosensor with TFET.

The ambipolar behavior of the TFET can be minimized with numerous approaches, and the overlapped gate over the drain is one kind of approach which yields good results for reduction of the ambipolar current. In the gate overlapping on the drain, the gate dielectric shows the impact on the widening of the energy bands at the channel-drain junction by increasing the gate oxide dielectric constant value, which leads to a reduction in the ambipolar current. The relation between the dielectric constant $(K)$ and the ambipolar current allows the detection of the targeted biomolecules by immobilizing in the nanogap created by the overlapped gate on the drain. The dielectric modulation technique made it an advantage for the biosensor design [11-15]. Here in this work, we demonstrated the advantage of the overlapped gate-on-drain in terms of the label-free biosensor by considering the nanowire gate all around-TFET (GAA-TFET).
Transport and tunneling of the charge carriers in TFET depend on two major factors; first, one is applied gate bias, and the second factor is how effectively the gate can control the channel. To inflate the gate controllability over the channel and enhance the tunneling rate, numerous techniques and gate engineering methods were introduced for TFET. The All-around gate is an engineered gate structure that yields excellent controllability over the intrinsic channel of TFET. The nanowire gate all around the TFET device induces superior controllability over the channel and reduce the leakage current $[16,17]$. The size of the target biomolecules in the given analyte is very small, and the more the surface to volume ratio acquired by the sensor will exhibit more sensitivity for the detection of the target biomolecules. Therefore, the nanowire-based biosensor can result in high sensitivity compared to the bulk device due to the increased surface to volume ratio attained by the nanowire-based biosensors and this high density of the biomolecules over the nanomaterial result in the experience in the high gating effect. There is colossal research that reported that the nanomaterial-based (nanoparticles, nanowires and nanotubes) biosensor results in increased sensitivity compared to the bulk devices [28, 35-37].

Here in this work, we have investigated the performance of nanowire GAA-TFET-based biosensor by considering the overlapped gate-on-drain technique. The partial hybridization and the practical binding difficulties of biomolecules are essential factors for biosensors, and here, we have included these effects to evaluate the sensitivity of the GAA-TFET biosensor. The entire work was carried out in four sections, and in section II, we discussed the architecture and the device's simulation strategies. Also, we elaborately described the device results and discussions in Section III by taking partial hybridization $(\mathrm{PH})$ and practical binding difficulties (PBD) of the biomolecule into consideration and the conclusion followed in the subsequent section IV.

\section{Architecture and simulation setup of GAA-TFET biosensor}

Figure 1 represents the 3D schematic arrangement of the proposed nanocavity curved overlapped gate-on-drain GAA-TFET biosensor. The 2D cross-sectional view of the proposed GAA-TFET biosensor provides an insight view of the proposed biosensor's spatial arrangement, which is illustrated in Fig. 2. The target biomolecules made immobilized in the nanocavity curved under the overlapped gateon-drain oxide. A $1 \mathrm{~nm}$ thick oxide layer $\left(\mathrm{SiO}_{2}\right)$ is used as an adhesion layer for the biomolecules immobilized in the nanocavity region under the overlapped gate-on-drain. This adhesion layer helps in strict binding of the biomolecules and immobilization in the nanocavity area. A single silicon 


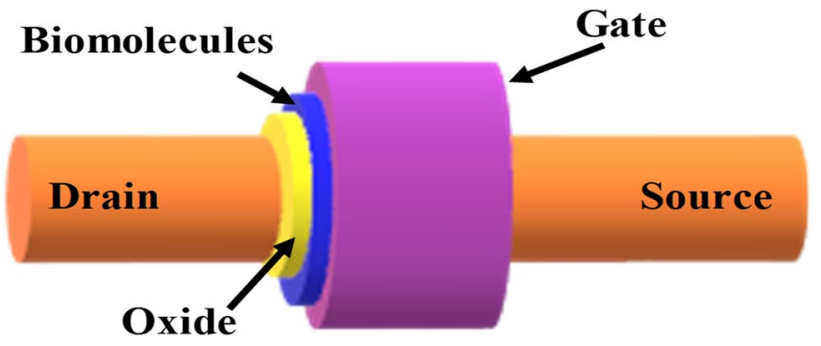

Fig. 1: 3D Schematic view of the proposed nanowire gate all aroundTFET (GAA-TFET) biosensor

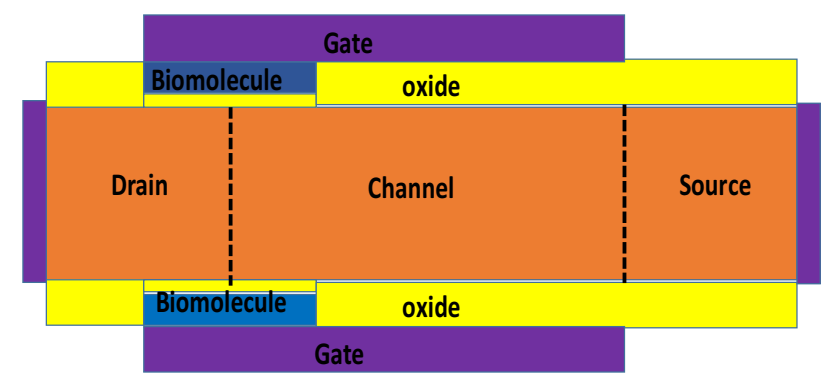

Fig. 2: 2D-Cross-sectional view of the nanowire gate all aroundTFET (GAA-TFET)-based biosensor with overlapped gate-on-drain

Table 1 Design specification and setup table for the proposed device

\begin{tabular}{ll}
\hline Design parameters & Value \\
\hline Gate length $\left(L_{G}\right) \mathrm{nm}$ & 70 \\
Drain length $\left(L_{D}\right) \mathrm{nm}$ & 50 \\
Source length, $\left(L_{S}\right) \mathrm{nm}$ & 50 \\
Radius of Si nanowire $(r) \mathrm{nm}$ & 10 \\
Thickness of the oxide, $\left(T_{\mathrm{ox}}\right) \mathrm{nm}$ & 4 \\
Overlapped length $\left(L_{\mathrm{ov}}\right) \mathrm{nm}$ & 40 \\
Thickness of the buffer oxide $\left(T_{\text {Box }}\right), \mathrm{nm}$ & 1 \\
Drain doping, $N$-Type, $\mathrm{cm}^{-3}$ & $5 \times 10^{18}$ \\
Channel doping, $(I), \mathrm{cm}^{-3}$ & $6 \times 10^{16}$ \\
Gate electrode work function & 4.8 \\
Source doping, $P$-type, $\mathrm{cm}^{-3}$ & $5 \times 10^{19}$ \\
\hline
\end{tabular}

nanowire is considered as the substrate for the biosensor design, and the simulation parameters are listed in Table 1.

Figure 3 represents the simulation structure of the proposed GAA-TFET biosensor with the immobilized biomolecules in the cavity region. The simulation of the device is carried out by introducing a material having a dielectric constant $(K>1)$ corresponding to the biomolecules (i.e., protein, biotin and streptavidin). The design parameters used for the GAA-TFET biosensor in this work is as follows: the radius of the silicon nanowire is $10 \mathrm{~nm}$, and the channel length is $70 \mathrm{~nm}\left(L_{\mathrm{ch}}\right)$, drain length is $50 \mathrm{~nm}\left(L_{D}\right)$, and the source length is taken as the $50 \mathrm{~nm}(L s)$. The source is doped with $p$-type $\left(1 \times 10^{19} \mathrm{~cm}^{-3}\right)$,

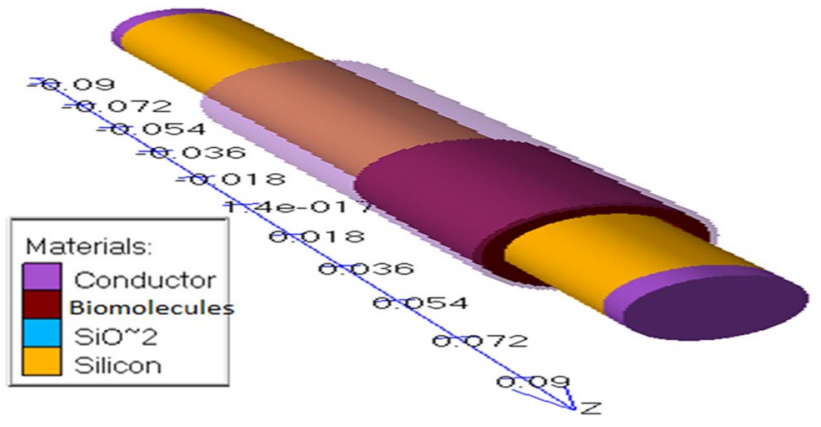

Fig. 3 The 3D simulated structure of GAA-TFET biosensor

the drain is with $n$-type $\left(5 \times 10^{18} \mathrm{~cm}^{-3}\right)$, and the channel is doped with $6 \times 10^{15} \mathrm{~cm}^{-3}$. The work function $(\varnothing)$ for the gate electrode is selected as 4.8 , and the thickness of the cavity is taken as $4 \mathrm{~nm}\left(T_{\text {cavity }}\right)$, and the overlapped-drain on gate length is taken as $50 \mathrm{~nm}\left(L_{\mathrm{ov}}\right)$. Table 1 represents the device parameters used for the simulation.

The device's simulation is performed by assuming that the cavity is filled by biomolecules entirely and made immobilized in the embedded nanocavity. The dielectric constant of the biomolecule in the overlapped gate-on-drain cavity is varied from $K=1$ to $K=10$ to investigate device performance under all circumstances. The benchmark reference value for measuring the sensitivity is competed filling the cavity with air having a dielectric constant value equal to $(K=1)$. The practical binding difficulties (PBD) and the partial hybridization (PH) effects of the biomolecules are taken into account to investigate the device's performance [19-22].

SILVACO Atlas, a commercially available TCAD simulator, is used for simulating the device structures [18]. The non-local BTBT (band-to-band tunneling) model is preferred for computing the tunneling probability of charge carrier at the interface; the Auger model, Shockley-Read-Hall recombination/generation model, Kane's model, the bandgap narrowing (BGN), the Fermi-Dirac carrier distribution models with default parameters are taken into consideration while doing the simulation of the device. The fabrication of the proposed device is complex compared to the conventional TFET devices, and the improved technology made the 3D device fabrication simple and feasible. Numerous researchers give many techniques to overcome these difficulties in device fabrication, and they are listed in works of literature with the modern nanolithography technology, etching and epitaxial growth techniques, which are fabricated into a real-time device [33-35].

\section{Results and discussions}

In this study, the ambipolar characteristics of the TFET are investigated immensely because the change in the ambipolar current (drain current $I_{d(\mathrm{amb})}$ ) of the TFET is taken 
as the sensing (s) parameter for detecting the presence of the target biomolecule. At first, we have made few assumptions before investigating the GAA-TFET biosensor that the nanogap under the overlapped gate on the drain is filled with biomolecules (i.e., protein, biotin, streptavidin and Uri case) and immobilized in the cavity. The dielectric constant of the immobilized biomolecules inside the cavity is varied in a range from $K=1$ to $K=10$, and the corresponding response in the ambipolar characteristics of the device noted down to analyze the device sensitivity as the dielectric constant of the biomolecule changes inside the nanocavity region results in the energy band bending near the drain-channel junction. Figure $4 \mathrm{a}$ shows the variation of the energy band diagram of the GAA-TFET biosensor in counter to the change in the dielectric constant of the biomolecule. Here, we changed the dielectric constant of the biomolecule form $K=1,2,3,5,7$, 10 , and it is observed from the corresponding energy band diagram that as the dielectric constant of the biomolecule is increases, it results in an increment in the bandgap at the channel-drain junction. The increment in the bandgap is linear up to $K=5$, and after the bandgap remains constant and, this happened because of the limitation of the potential inside the nanowire GAA-TFET biosensor. If the dielectric constant value of the biomolecule is further increased beyond $K=10$, the energy bandgap of the device remains stable due to fringing field effects given by surrounded gate structure.

From Fig. 4a, it is observed that the bandgap increases with increasing the dielectric constant of the biomolecule, and due to this increased energy bandgap, the tunneling width of the charge carrier at the channel-drain junction increases and results in the reduction of the ambipolar current of the device. The corresponding ambipolar current for the change in the dielectric constant of biomolecules is shown in Fig. 4b. The transfer characteristics of the overlapped gate-on-drain GAA-TFET biosensor for different dielectric constant values of the biomolecules ranging from $K=1$ to $K=5$ are shown in Fig. $4 \mathrm{~b}$. Here, we represented the absence of the biomolecule by taking a new material with dielectric constant $K=1$ (Air), and this is taken as the reference for detecting the presence of biomolecule and measuring the device's sensitivity. The mathematical formulation of the sensitivity of the device is expressed in Eq. 1.

Sensitivity $(S)=\frac{I \mathrm{ds}(\text { air })}{I \mathrm{ds}(\text { bio })}$

For the dielectric modulation-based biosensor, the biomolecule's polarity either improves or decreases the sensitivity for a low dielectric constant $(K)$ biomolecules depends on the types of Si substrate doping where the cavity is created. Here, we considered the overlapped gate-on-drain technique where the sensitivity depends on the drain depletion and the higher the drain depletion, the higher the sensitivity. The charge of the biomolecule can show the notable impact on sensitivity for low dielectric constant valued biomolecules. However, when the dielectric constant of the biomolecule increases, it will dominate and reduce the charge of the biomolecule on sensitivity [23-27].

Figure 5a represents the proposed biosensor's transfer characteristic for the dielectric constant values $K=5,7$, and 10. From this Fig. 5a, we observed that even the dielectric

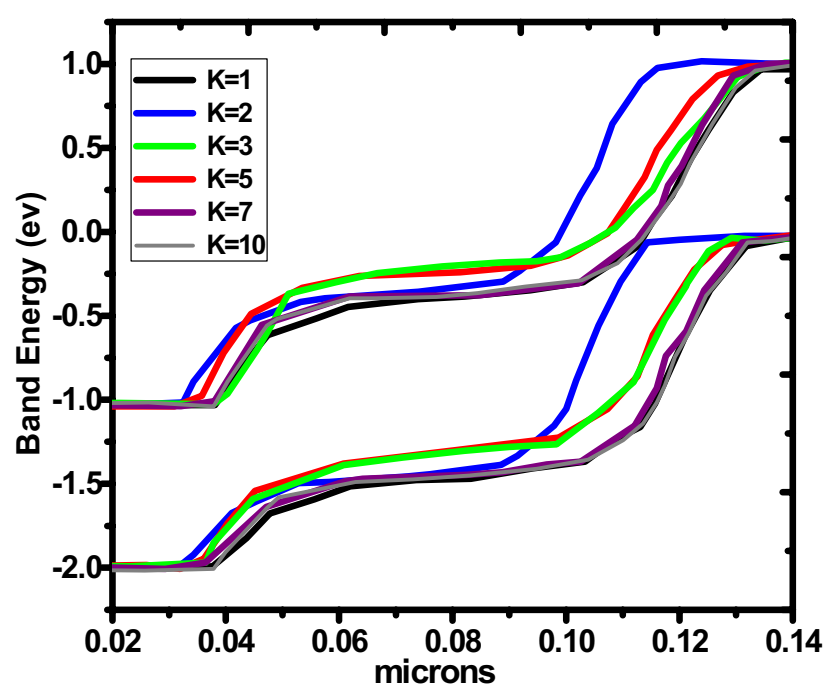

Fig. 4 a: Energy band diagram of the nanowire gate all around-TFET (GAA-TFET)-based biosensor with overlapped gate-on-drain for dielectric value from $K=1$ to 5 for $V_{\mathrm{ds}}=0.5 \mathrm{~V}$ b Transfer character-

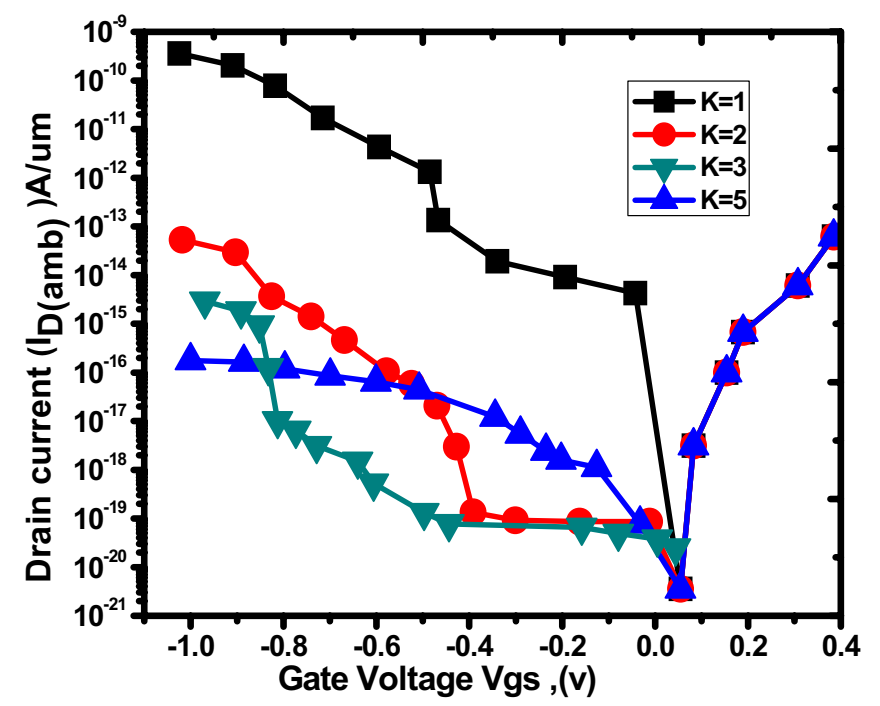

istics of nanowire gate all around-TFET (GAA-TFET)-based biosensor with overlapped gate-on-drain with increasing dielectric constant from 1 to 5 and $V_{\mathrm{ds}}=0.5 \mathrm{~V}$. 

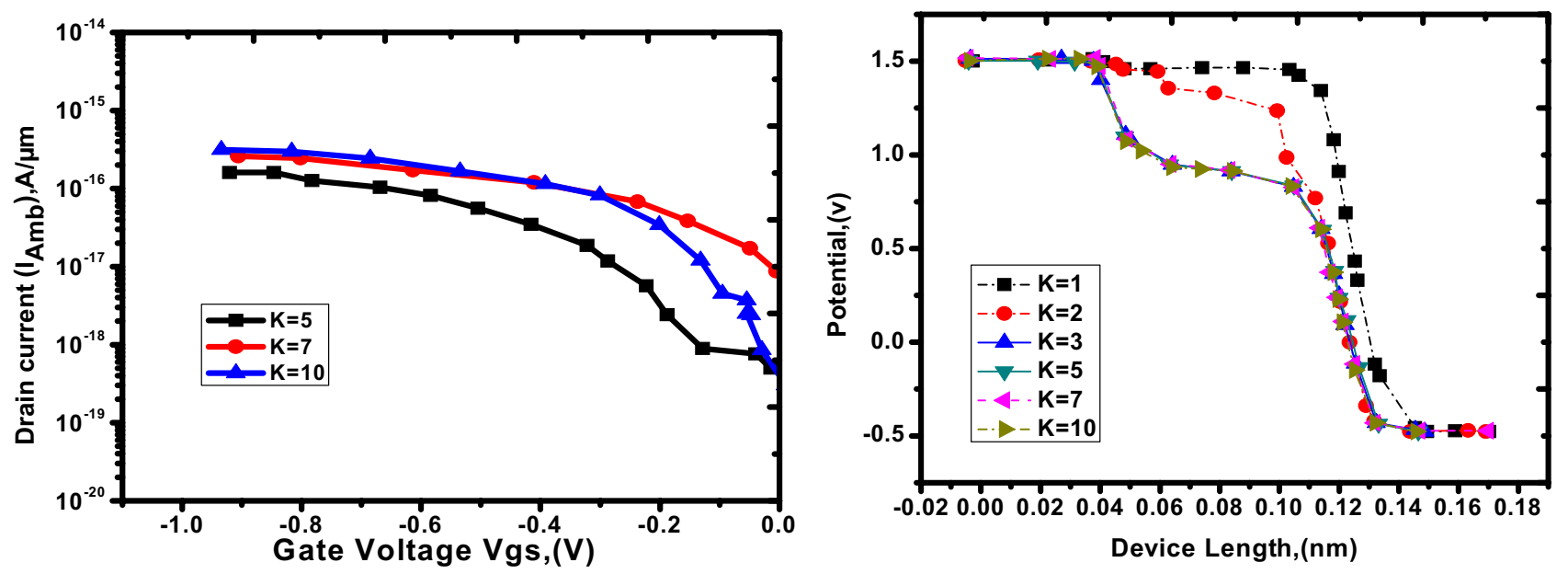

Fig. 5 a Shows the transfer characteristics of nanowire gate all around-TFET (GAA-TFET)-based biosensor with overlapped gate-on-drain with dielectric constant $K=5,7,10$, b Potential variation along the device length with the different dielectric constant of biomolecules

constant value is doubled, it shows a little variation in the ambipolar current, which is neglected and treated as constant. This is due to the effect of the energy band profile modulation (BPM), where the further widening of the bandgap is not possible because of the limitation on the minimum doping profile of the drain [27-31]. Even the surrounded gate structure shows high controllability over the channel, but the technique overlapping gate-on-drain depends on drain doping concentration. Here, we considered the drain doping of $5 \times 10^{18}$, which cannot further reduce due to the sensor's construction constraints [27-31]. Figure 5b represents the potential variation of the GAA-TFET biosensor for different dielectric constant values of the biomolecules. The increase in the value of the dielectric constant of the biomolecule in the nanocavity influence the inter-trapped charge carriers near the channel-drain junction. These inter-trapped charge carriers create a potential change in the nanowire semiconductor, and as a result, the band starts changing their position.

The horizontal electric field along the device is shown in Fig. 6 for the targeted biomolecules with different dielectric constant values. Figure 6 shows that the overlapped gate-on-drain region possesses a high electric field near the channel-drain junction than the source-channel junction due to biomolecule presence under the cavity region of the overlapped gate-on-drain. This positive effect further increases the depletion width at the channel-drain junction and reduces ambipolar transport. The generalized expression for measuring the device's sensitivity is expressed by Eq. 1, and the corresponding values are listed in Table 2. The measured sensitivity values from Table 2 shows that the proposed biosensor's sensitivity increases linearly with the dielectric constant value up to $K=5$ and, for higher dielectric values of biomolecules, i.e., $K>5$, there is a slight downfall of

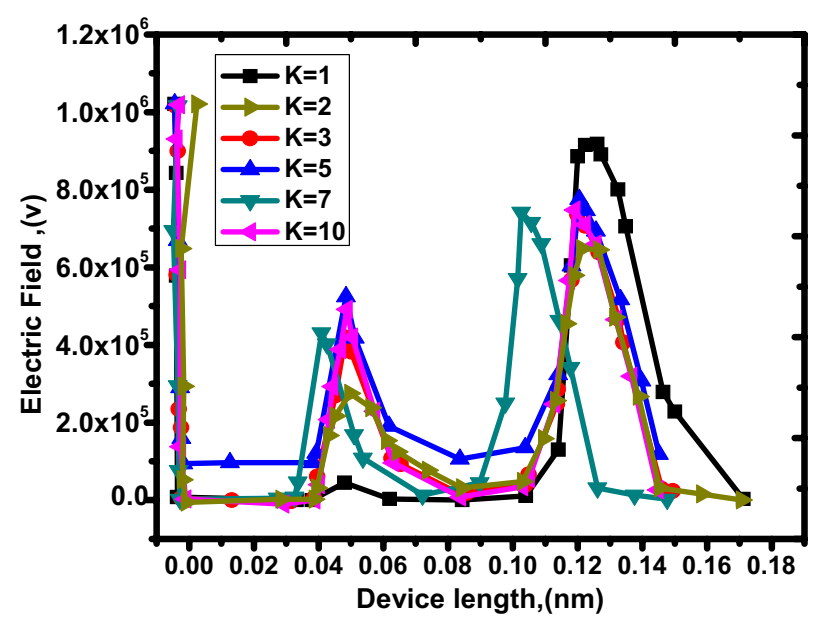

Fig. 6 Variation of the electric field for different dielectric constant values of biomolecules with $V_{\mathrm{gs}}=1 \mathrm{v}$, and $V_{\mathrm{ds}}=0.5 \mathrm{v}$

Table 2 Sensitivity variation with dielectric constant value of the biomolecules

\begin{tabular}{ll}
\hline $\begin{array}{l}\text { Dielectric constant of the Target biomol- } \\
\text { ecules }\end{array}$ & Sensitivity $(S)=\frac{I d s(\text { air })}{I d s(\text { bio })}$ \\
\hline $\mathrm{K}=1$ & 1 \\
$\mathrm{~K}=2$ & $6.73 \times 10^{3}$ \\
$\mathrm{~K}=3$ & $1.22 \times 10^{5}$ \\
$\mathrm{~K}=5$ & $2.03 \times 10^{6}$ \\
$\mathrm{~K}=7$ & $1.92 \times 10^{6}$ \\
$\mathrm{~K}=10$ & $1.90 \times 10^{6}$ \\
\hline
\end{tabular}

sensitivity which is negligible and treated as constant. For the higher dielectric constant value of the biomolecules, the 
nanowire GAA-TFET shows no variation in the sensitivity because of the drain doping limitation [27-32]. The bandgap cannot increase further near the channel-drain junction even at the higher dielectric constant value of the biomolecules.

Figure 7 represents the drain current sensitivity plot of the nanowire GAA-TFET biosensor with overlapped gateon-drain. The proposed biosensor can express the sensitivity, which the ratio of drain current without biomolecule (i.e., $k=1$ ) to with the presence of biomolecule, with high sensitivity $10^{6}$ for a wide range of biomolecules (i.e., $K=5$ ) and it maintained to all the higher dielectric constant values of the biomolecules.

The nanowire GAA-TFET biosensor shows excellent improvement in the device sensitivity in terms of high drain current variation. Apart from this, the nanowire GAA-TFET

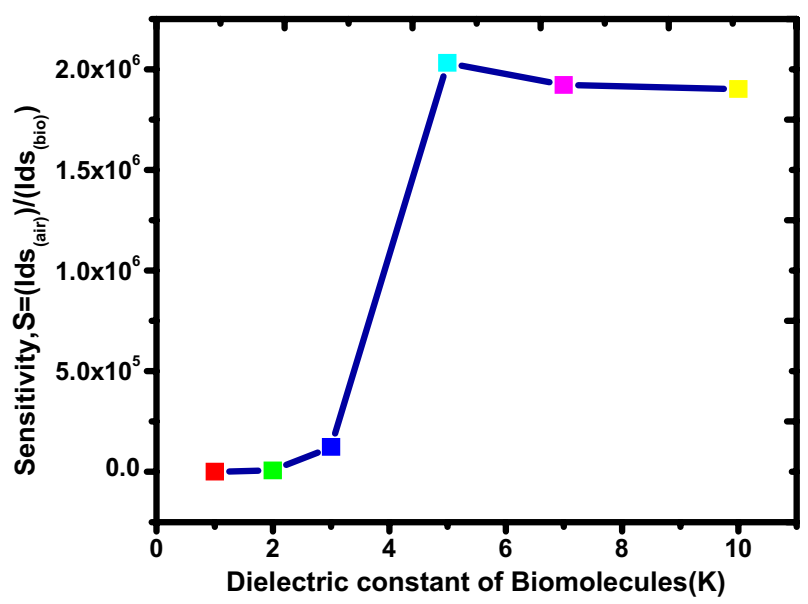

Fig. 7 Sensitivity $I_{\mathrm{ds}}\left(\left(_{\text {air }} / I_{\mathrm{ds}(\text { bio })}\right.\right.$ for different biomolecules with $V_{\mathrm{gs}}=-$ $1 \mathrm{v}$ and $V_{\mathrm{ds}}=0.5 \mathrm{v}$

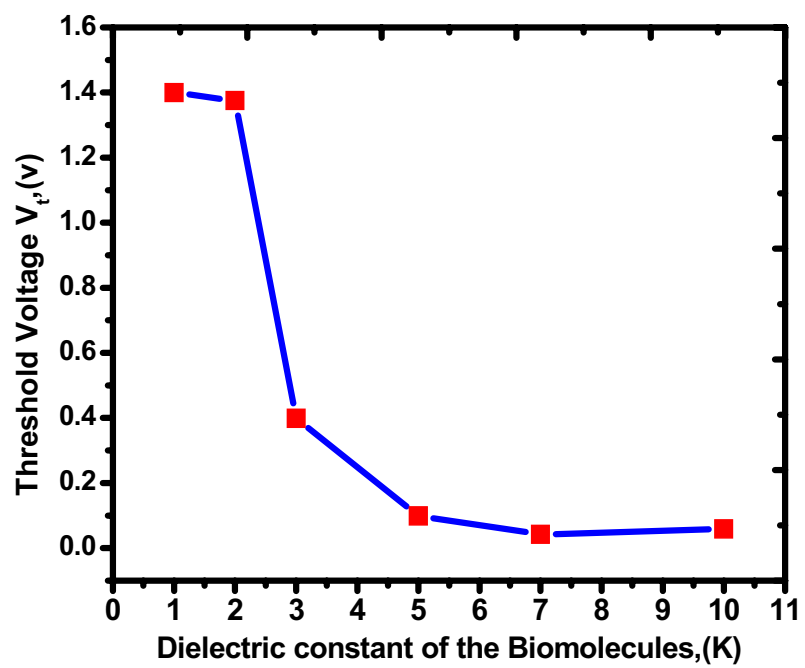

biosensor is capable of exhibiting high, prominent threshold voltage characteristics [22-32]. The device's surrounded gate structure facilitates the gate with high controllability over the intrinsic channel and makes it operate at low voltages. Figure 8a represents the threshold voltage variation of the proposed nanowire GAA-TFET biosensor for different biomolecules. It is observed in Fig. 8a that the increment in the dielectric constant value of immobilized biomolecule inside the nanocavity reduces the threshold voltage of the device to a greater extent, and this is due to the increment of the overlapped gate capacitance near the channel-drain junction and reduced leakage current. We have taken the variation in the device's threshold voltage as the parameter for detecting the biomolecule. The threshold voltage sensitivity is plotted in Fig. $8 \mathrm{~b}$ using the following mathematical relation.

Sensitivity $\left(S_{V \text { th }}\right)=(V \operatorname{th}($ air $)-V \operatorname{th}($ bio $)) / V$ th(air)

All results discussed in this section are performed by assuming that the nanogap region under the overlapped gate is filled completely with biomolecules. However, this case is impossible due to the biomolecules associated binding difficulties and the fill factor. Partial hybridization (PH) is one of the essential factors to be considered when designing a biosensor, and this effect occurs due to incomplete filling of the cavity and the steric hindrance effect of the biomolecules. Many people deliberately discussed these $\mathrm{PH}$ effects and the binding difficulties to realize the biosensor's real-time performance [19-22].

Here we have incorporated the biomolecules' binding difficulties by partial filling the cavity with air in the nanocavity

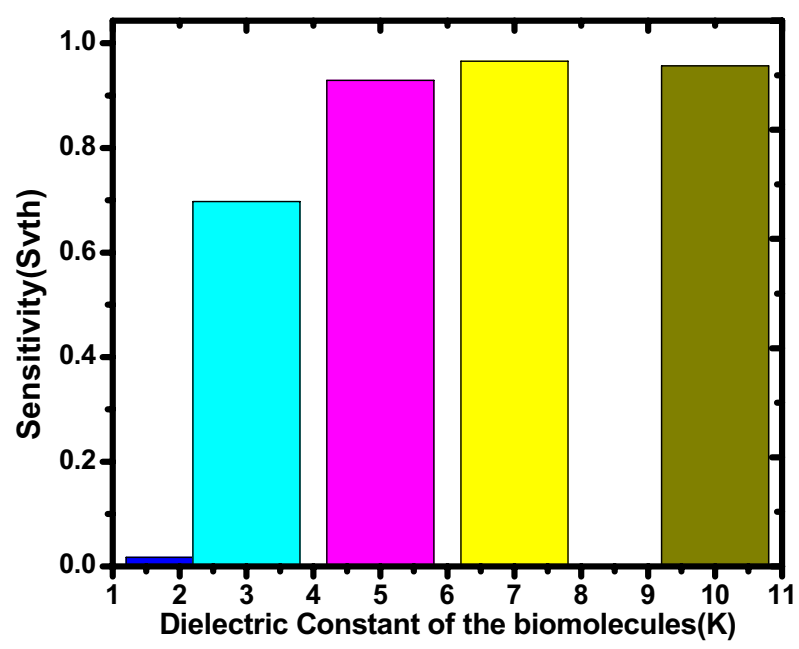

Fig. 8 a Threshold voltage variation of the nanowire GAA-TFET biosensor for the different dielectric constant of biomolecules. b Threshold voltage sensitivity of the nanowire GAA-TFET biosensor 


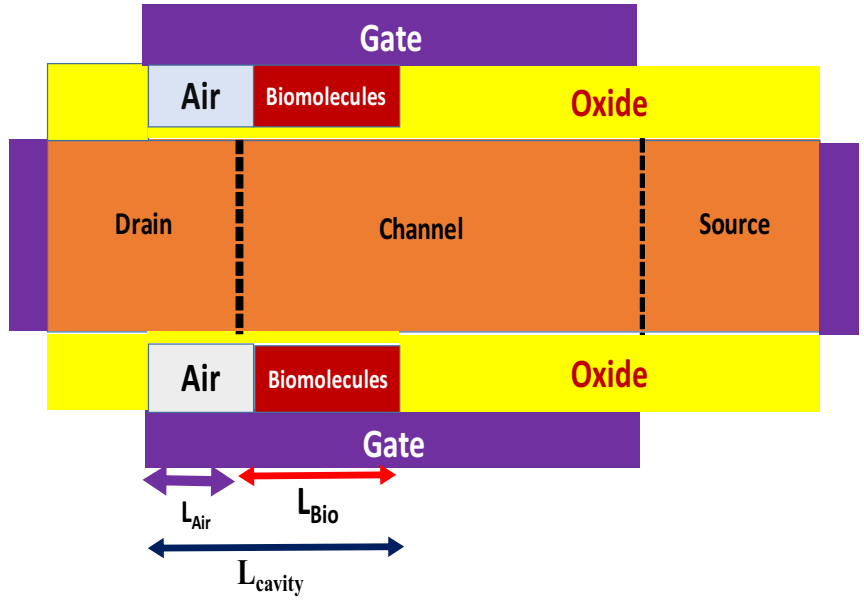

(a)

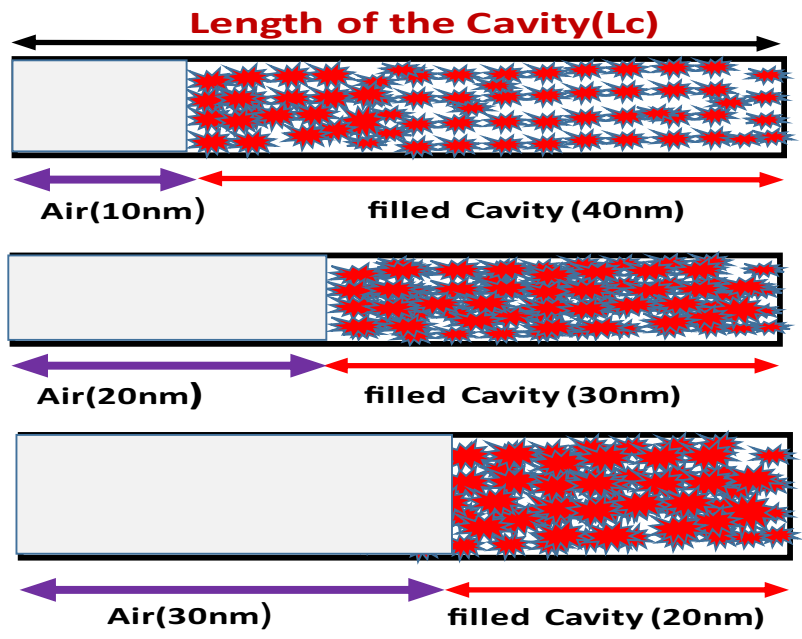

(b)

Fig. 9 a 2D Schematic view of the nanowire GAA-TFET biosensor with the partially filled cavity b Different cases of filling he nanocavity with air under the overlapped gate $(10 \mathrm{~nm} .20 \mathrm{~nm}, 30 \mathrm{~nm})$ and $K=5$ for the biomolecules

\section{Conclusion}

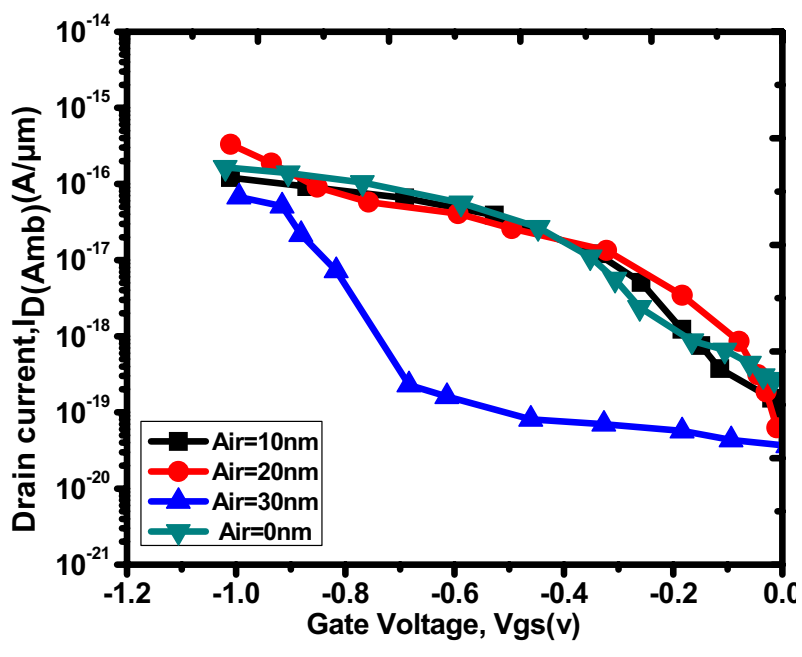

Fig. 10 Transfer characteristic of the proposed biosensor by considering fill factor and Partial Hybridization (PH) effect

region. Here we have taken three cases where the air can occupy the nanogap into different length, which is showed in Fig. 9. (b), i.e. $10 \mathrm{~nm}, 20 \mathrm{~nm}$ and $30 \mathrm{~nm}$. For all three cases, the partially filled biomolecules dielectric constant is taken as constant, i.e. $K=5$, and we have noted down the respective drain current of the biosensor for all three cases, and the corresponding drain currents are plotted in the Fig. 10. Interestingly the $\mathrm{PH}$ and the binding problems associated with the biomolecules cannot show an impact on the device performance due to high controllability of the gate over the channel.
This paper presented a GAA-TFET-based biosensor using the dielectric modulation (DM) technique by placing the biomolecules inside the nanocavity curved in the oxide dielectric overlapped gate-on-drain region. Here, we used the nanowire GAA-TFET device for getting higher controllability over the channel, which yields higher sensitivity. It is observed that the device shows a variation of $10^{3}$ amount of drain current $\left(\boldsymbol{I}_{\mathbf{d s}(\mathbf{a m b})}\right)$ by increasing the dielectric $(K)$ constant value of the biomolecules from 1 to 5 . It is also concluded that ambipolar transport, which is the drawback of the TFET for digital circuit applications, turns into an advantage for biosensor applications. The excellent and remarkable performance of the proposed GAA-TFET-based biosensor made it an advancement in the field of biosensors. In the future, this will play a vital role in the detection of biomolecules with controlled gate leakage current with superior low power functionality.

Acknowledgements The authors acknowledge VIT-AP university for providing the necessary facilities for carrying out the research work.

\section{Declarations}

Conflict of interest The authors declare that they have no conflict of interest.

Consent for publication The authors have given Consent for Publication as per the journal policy.

Ethical approval The manuscript is prepared as per the ethical standard of the journal. 


\section{References}

1. P. Mehrotra, ScienceDirect biosensors and their applications - a review. J. Oral Biol. Craniofacial Res. 2015, 1-7 (2016)

2. R. Esfandyarpour, M. Javanmard, Z. Koochak, H. Esfandyarpour, J.S. Harris, R.W. Davis, Label-free electronic probing of nucleic acids and proteins at the nanoscale using the nanoneedle biosensor. Biomicrofluidics, 7(4), 044114 (2013)

3. Jr. Clark, C. Leland, Champ Lyons, "Electrode systems for continuous monitoring in cardiovascular surgery." Annals of the New York Academy of sciences 102(1), 29-45 (1962)

4. V. Hemaja, D.K. Panda, A comprehensive review on high electron mobility transistor (HEMT) Based biosensors : recent advances and future prospects and its comparison with Si-based biosensor. Silicon (2021). https://doi.org/10.1007/s12633-020-00937-w

5. K.I. Chen, B.R. Li, Y.T. Chen, Silicon nanowire field-effect transistor-based biosensors for biomedical diagnosis and cellular recording investigation. Nano Today 6(2), 131-154 (2011)

6. J.-M. Choi, J.-W. Han, S.-J. Choi, Y.-K. Choi, Analytical modeling of a nanogap-embedded FET for application as a biosensor. IEEE Trans. Electron Devices 57(12), 3477-3484 (2010)

7. H. Im, X.-J. Huang, B. Gu, Y.-K. Choi, A dielectric-modulated field-effect transistor for biosensing. Nat. Nanotechnol. 2(7), 430-434 (2007)

8. D. Sarkar, K. Banerjee, Fundamental limitations of conventional-FET biosensors: Quantum-mechanical-tunneling to the rescue. Device Res Conf-ConfDig DR C 83-84 (2012)

9. R. Narang, M. Saxena, M. Gupta, Comparative Analysis of Dielectric-Modulated FET and TFET-Based Biosensor. IEEE Trans. Nanotechnol. 14(3), 427-435. https://doi.org/10.1109/ TNANO.2015.2396899

10. N.N. Reddy, D.K. Panda, A comprehensive review on tunnel field-effect transistor (TFET) based biosensors: recent advances and future prospects on device structure and sensitivity. Silicon (2020). https://doi.org/10.1007/s12633-020-00657-1

11. D.B. Abdi, M.J. Kumar, S. Member, Controlling ambipolar current in tunneling FETs using overlapping gate-on. IEEE J. Electron Devices Soc. 2(6), 28-31 (2014)

12. N.N. Reddy, D.K. Panda, Simulation study of dielectric modulated dual material gate TFET based biosensor by considering ambipolar conduction. Silicon (2020). https://doi.org/10.1007/ s12633-020-00784-9

13. S. Garg, S. Saurabh, Suppression of ambipolar current in tunnel FETs using drain-pocket: proposal and analysis. Superlattices Microstruct. 113, 261-270 (2017)

14. D.B. Abdi, M.J. Kumar, Superlattices and microstructures dielectric modulated overlapping gate-on -drain tunnel-FET as a label-free biosensor. Superlattices Microstruct. 86, 198-202 (2015). https://doi.org/10.1016/j.spmi.2015.07.052

15. R. Narang, M. Saxena, M. Gupta, Ambipolar behaviour of tunnel field effect transistor (TFET) as an advantage for biosensing applications. Phys. Semiconductor Devices 171-172. (2014). https://doi.org/10.1007/978-3-319-03002-9

16. Ajay, M. Gupta, R. Narang, M. Saxena, "Analysis of GaSb-InAs Gate all around (GAA) p-i-n tunnel FET (TFET) for application as a bio-sensor,". In: 2016 IEEE International nanoelectronics conference (INEC), Chengdu, pp. 1-2, (2016). https://doi.org/ 10.1109/INEC.2016.7589324.

17. N. Shafi, J.S. Parmaar, A. Porwal, A.M. Bhat, C. Sahu, \& C. Periasamy, Gate All around junctionless dielectric modulated biofet based hybrid biosensor: design, simulation and performance investigation. Silicon. (2020). https://doi.org/10.1007/ s12633-020-00583-2
18. ATLAS (2012) Device simulation software. Silvaco Int, Santa Clara

19. A. Bhattacharyya, M. Chanda, D. De, "Performance assessment of new dual-pocket vertical heterostructure tunnel FET-based biosensor considering steric hindrance issue.” IEEE Trans Electron Devices 66, 3988-3993 (2019)

20. R. Narang, M. Saxena, M. Gupta, Comparative analysis of dielectric-modulated FET and TFET-based biosensor. IEEE Trans. Nanotechnol. 14(3), 427-435 (2015)

21. A. Bhattacharyya, M. Chanda, D. De, Analysis of partial hybridization and probe positioning on sensitivity of a dielectric modulated junctionless label free biosensor. IEEE Trans. Nanotechnol. 19, 719-727 (2020). https://doi.org/10.1109/TNANO.2020.30255 44

22. R. Goswami, B. Bhowmick, Comparative analyses of circular gate TFET and heterojunction TFET for dielectric-modulated labelfree biosensing. IEEE Sens. J. 19(21), 9600-9609 (2019). https:// doi.org/10.1109/JSEN.2019.2928182

23. K. Han, S. Long, Z. Deng, Y. Zhang, J. Li, A novel germaniumaround-source gate-all-around tunnelling field-effect transistor for low-power applications. Micromachines 11(2), 164 (2020). https://doi.org/10.3390/mi11020164

24. U. Dutta, M.K. Soni, M. Pattanaik, Design \& optimization of gate-all-around tunnel FET for low power applications. Int. J. Eng. Technol. UAE 7(4), 2263-2270 (2018). https://doi.org/10.14419/ ijet.v7i4.12352

25. A. Singh, S. Chaudhury, C.K. Pandey, S.M. Sharma, C.K. Sarkar, Design and analysis of high $\mathrm{k}$ silicon nanotube tunnel FET device. IET Circuits Devices Syst. 13(8), 1305-1310 (2019). https://doi. org/10.1049/iet-cds.2019.0230

26. A. Ravindran, A. George, C.S. Praveen, N. Kuruvilla, Gate all around nanowire TFET with high ON/OFF current ratio. Mater. Today: Proceed. 4(9), 10637-10642 (2017). https://doi.org/10. 1016/j.matpr.2017.06.434

27. C.H. Kim, C. Jung, H.G. Park, Y.K. Choi, Novel dielectric-modulated field-effect transistor for label-free DNA detection. BioChip J. 2(2), 127-134 (2009)

28. X.P.A. Gao, G. Zheng, C.M. Lieber, Subthreshold regime has the optimal sensitivity for nanowire FET biosensors. Nano Lett. 10(2), 547-552 (2010). https://doi.org/10.1021/n19034219

29. N.N. Reddy, D.K. Panda, Performance analysis of Z-shaped gate dielectric modulated (DM) tunnel field-effect transistor-(TFET) based biosensor with extended horizontal N+pocket. Int. J. Numer Model (2021). https://doi.org/10.1002/jnm.2908

30. D.B. Abdi, M.J. Kumar, Controlling ambipolar current in tunneling FETs using overlapping gate-on-drain. IEEE J. Electron Devices Soc. 2(6), 187-190 (2014). https://doi.org/10.1109/JEDS. 2014.2327626

31. T. Krishnamohan, D. Kim, S. Raghunathan, K. Saraswat, Double-gate strained-Ge heterostructure tunneling FET (TFET) with min, Ioff on-state ambipolar-state simulation (Quantum transport). Simulation 67, 947-949 (2008). https://doi.org/10.1109/IEDM. 2008.4796839

32. J.S. Lee, J.H. Seo, S. Cho, J.H. Lee, S.W. Kang, J.H. Bae, E.S. Cho, I.M. Kang, Simulation study on effect of drain underlap in gate-all-around tunneling field-effect transistors. Curr. Appl. Phys. 13(6), 1143-1149 (2013). https://doi.org/10.1016/j.cap.2013.03. 012

33. C.K. Pandey, D. Dash, S. Chaudhury, Impact of dielectric pocket on analog and high-frequency performances of cylindrical gateall-around tunnel FETs. ECS J. Solid State Sci. Technol. 7(5), N59-N66 (2018). https://doi.org/10.1149/2.0101805jss 
34. V.B. Sreenivasulu, V. Narendar, Performance improvement of spacer engineered n-type SOI FinFET at 3-nm gate length. AEUInt. J. Electro. Commun. 137, 153803 (2021)

35. M. Kumar, Y. Pratap, S. Haldar, M. Gupta, R.S. Gupta, Cylindrical gate all around Schottky barrier MOSFET with insulated shallow extensions at source / drain for removal of ambipolarity : a novel approach. J. Semicond. (2017). https://doi.org/10.1088/ $1674-4926 / 38 / 12 / 124002$

36. E. Stern, J.F. Klemic, D.A. Routenberg, P.N. Wyrembak, D.B. Turner-Evans, A.D. Hamilton, D.A. LaVan, T.M. Fahmy, M.A. Reed, Label-free immunodetection with CMOS-compatible semiconducting nanowires. Nature 445(7127), 519-522 (2007). https:// doi.org/10.1038/nature05498 (PMID: 17268465)
37. J.A. Streifer, H. Kim, B.M. Nichols, R.J. Hamers, Covalent functionalization and biomolecular recognition properties of DNAmodified silicon nanowires. Nanotechnology 16, 1868 (1868). https://doi.org/10.1088/0957-4484/16/9/075

Publisher's Note Springer Nature remains neutral with regard to jurisdictional claims in published maps and institutional affiliations. 